\title{
Parameters Affecting the Erosive Burning of Solid Rocket Motor
}

\author{
Almostafa Abdelaziz', Liang Guozhu' ${ }^{1}$ and Anwer Elsayed ${ }^{2}$ \\ ${ }^{1}$ Beihang University (BUAA), Aerospace Department, 100191 Beijing, China \\ ${ }^{2}$ Militery Technical College (MTC), Rocket Department, Cairo, Egypt
}

\begin{abstract}
Increasing the velocity of gases inside solid rocket motors with low port-to-throat area ratios, leading to increased occurrence and severity of burning rate augmentation due to flow of propellant products across burning propellant surfaces (erosive burning), erosive burning of high energy composite propellant was investigated to supply rocket motor design criteria and to supplement knowledge of combustion phenomena, pressure, burning rate and high velocity of gases all of these are parameters affect on erosive burning. Investigate the phenomena of the erosive burning by using the 2'inch rocket motor and modified one. Different tests applied to fulfil all the parameters that calculated out from the experiments and by studying the pressure time curve and erosive burning phenomena.
\end{abstract}

\section{Introduction}

The erosive burning refers to the increase in the propellant burning rate caused by the high-velocity flow of combustion gases over the burning propellant surface[1]. It can seriously affect the performance of solid propellant motors. It occurs primarily in the port passages or perforations of the grain as the combustion gases flow toward the nozzle [2]. It is more probable to occur when the port passage cross-section area is low relative to the throat area with a port-to-throat field [3].

Erosive burning increases the gas mass flow and thus also the chamber pressure and thrust during the early portion of the burning, for a particular motor, as soon as the burning enlarges the flow passage (without a major increase in burning area), the port area flow velocity is reduced and erosive burning diminishes until normal burning will again occur. Erosive burning causes also early burn out of the web usually at the nozzle end and lets out the insulation and ceasing to hot combustion gas for a large period of the fourth dimension. This usually needs more insulation layer thickness (and more inert mass) to prevent local thermal failure[4,5].

A relatively simple model for erosive burning based on heat transfer was first developed in 1956 by LenoirRobillard [6] and has since been improved and used in motor performance calculations. The model has the simplified form.

In this paper we present some factors which affect the erosive burning phenomena, and by coding a MATLAB code to investigate the internal ballistic of the rocket motor. Some experiments will take part for describe the phenomena of erosive burying which takes part in small 2 inch rocket motor and then by modified solid rocket motor which shows clearly the phenomena of the erosive burning.

\section{Factors Affecting Erosive Burning}

Cross-flow velocity, pressure, propellant initial temperature, free stream gas temperature, normal burning rate, metal addition, compressibility, oxidizer particle size, rocket motor size, propellant characteristic, mass flux and Reynolds number. All of this parameters can affect the erosive burning $[7,8]$, we will describe the effect of total length to port area diameter to describe the phenomena by MATLAB code for investigating the internal ballistic and by some sets of experiments to show the phenomena clearly by different designs of solid rocket motor. A relatively simple model for erosive burning based on heat transfer was first developed by Lenoir-Robillard. The model has the simplified form.

$$
r_{t}=r_{o}+r_{e}
$$

Where $r_{t}$ is the total burning rate, $r_{0}$ is the base burning rate and $r_{e}$ is the burning due to erosive burning rate.

$$
\begin{aligned}
& r_{t}=a p^{n}+\alpha G^{.0 .8} L^{-0.2} e^{\left(\frac{-\beta^{*} t_{t}^{*} \rho^{*} p}{G}\right)} \\
& G=\frac{m}{A_{p}} \\
& r_{t}=a p^{n}+\alpha G^{.0 .8} d^{-0.2} e^{\left(\frac{-\beta^{*} t_{t}^{*} \rho^{*} p}{G}\right)}
\end{aligned}
$$


Where equation (2) used when depending on the length of the propellant and equation (3) when use the inner diameter of the propellant. (Fig. 1) shows the effect of erosive burning. Which show the deviation of the course of pressure variation with time due to erosive burning. where $\alpha$ is the erosive burning coefficient, $G$ is the mass flux, $\mathrm{L}$ is the initial grain length, $\beta$ is the erosive burning pressure coefficient, and $\rho_{p}$ is the propellant density, Ap is the port area and, $d$ is the inner diameter of the grain.

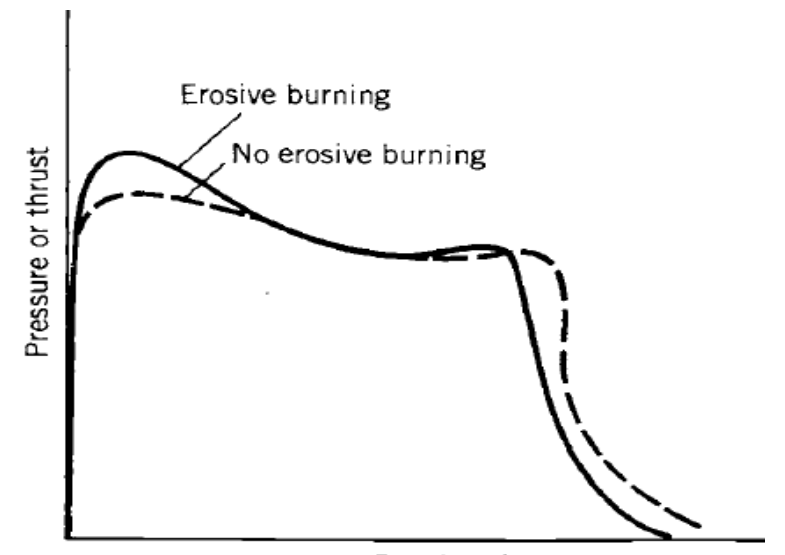

Burning time

Figure 1. Erosive burning phenomena.

\section{Experiments of 2 inch motors}

The main task of the test motor is to determine the burning rate of the propellant, combustion index and burning rate coefficient calculated by a fitting curve to determine the burning law. A tubular grain from composite propellant is cast inside a special casing so that the burning is allowed only from inside and from the two ends. The main output of the test is the pressure timecurve.

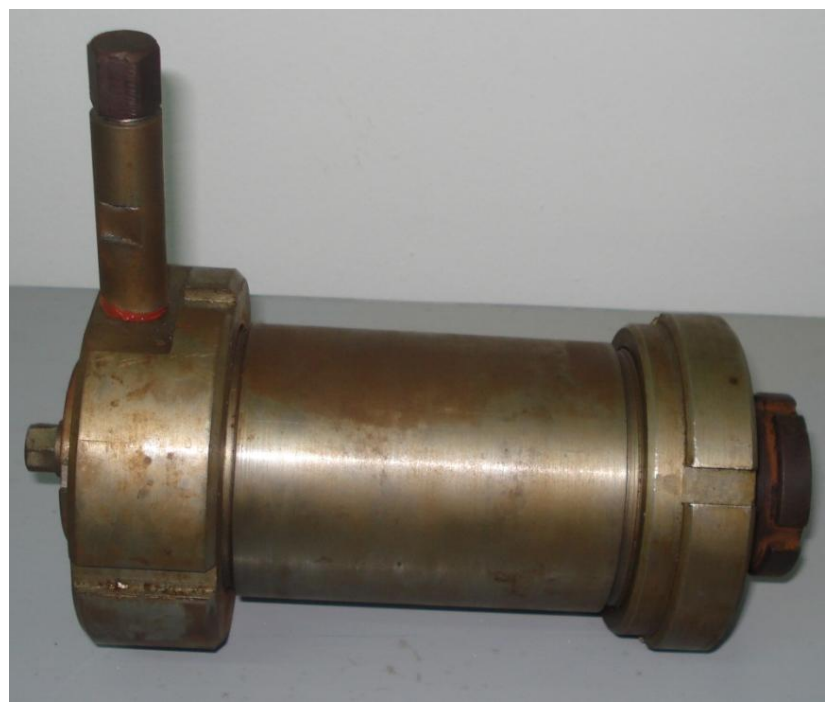

Figure 2. 2 inch motor.

From the main parameters that affect the erosive burning, decrease the port diameter of the grain by making a modified grain with different port diameter 34 ,
$15,12.5$ and $10 \mathrm{~mm}$ respectively to show the effect of the decreased diameter with the same length $108 \mathrm{~mm}$. By decreasing the port diameter the velocity of gases parallel to the burning surface increased, which causes erosive burning in the propellant $[9,10]$, by using composite propellant consist of ammonium Perchlorate (68-70\%) aluminum powder (16-18\%) and binder (13-15\%).

\section{The results of the tests}

Table 1. Output data with different port diameters.

\begin{tabular}{|l|l|l|l|l|l|}
\hline D & $\mathrm{mm}$ & 34 & 15 & 12.5 & 10 \\
\hline Temp & $\mathrm{c}$ & 21 & 21 & 21 & 21 \\
\hline Throat & $\mathrm{mm}$ & 7 & 7 & 7 & 7 \\
\hline WT & Kg & .375 & .512 & .503 & .514 \\
\hline TD & Msec & 20 & 12 & 10 & 387 \\
\hline TB & Msec & 2.24 & 4.06 & 4.31 & 4.43 \\
\hline T50\% & Sec & 2.13 & 3.9 & 4.1 & 4.32 \\
\hline Pmax & Bar & 71 & 86 & 55 & 74 \\
\hline IPT & Bar.s & 151 & 199 & 183 & 197 \\
\hline Pav & Bar & 67 & 48 & 42 & 43 \\
\hline P50\% & Bar & 71 & 50 & 43 & 45 \\
\hline R50\% & mm/s & 6.1 & 5.7 & 5.6 & 5.7 \\
\hline Cstar & m/sec & 1553 & 1498 & 1401 & 1475 \\
\hline
\end{tabular}

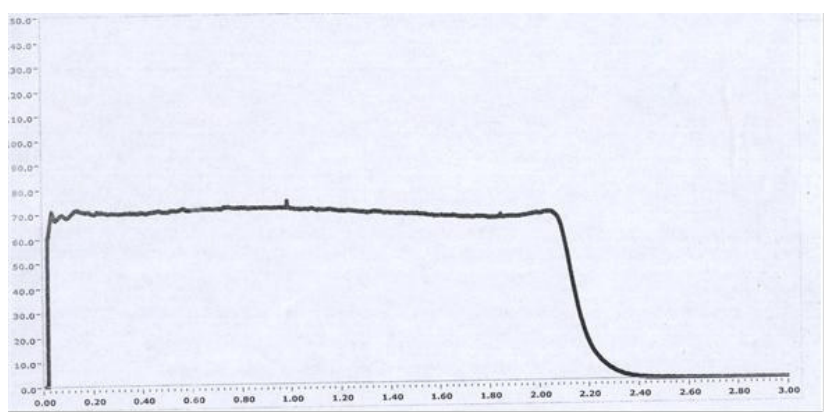

Figure 3. pressure time curve at port diameter $34 \mathrm{~mm}$.

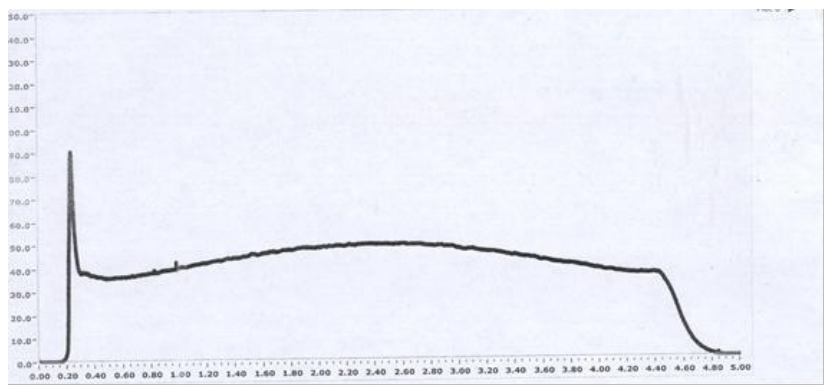

Figure 4. pressure time curve at port diameter $10 \mathrm{~mm}$.

This increase in the beginning of the propellant burning is due to using the greater mass of the black powder. 
From the calculation of the parameters of the test and Thermochemical calculation when decreaseing the port diameter and make the critical diameter constant the velocity of gases increase from 37 to $300 \mathrm{~m} / \mathrm{s}$, and the erosive ratio from 1 to 1.36 .

This information is not enough to investigate the phenomena of erosive burning. In other tests shown later, the length of the rocket motor will be increased to investigate the phenomena of erosive burning clearly.

\section{Computer program}

The computer program is capable of calculating performance prediction of tabular grain and draw pressure time curve, the program computes in SI units which are used to calculate the main parameters and performance of solid propellant rocket motor, the program may be used for rocket of all size, the main task of the program is to draw the P-T curve and to calculate and the phenomena of the erosive burning.

We will divide the web thickness into equal segments for easy calculation and determine the output with minimum error.

The internal ballistic analysis follow zero dimensional frictionless compressible flow theory with the flow taken as isentropic in the nozzle, equilibrium pressures are established by iteration to find the correct chamber pressure which balance the continuity equation. By dividing the web thickness into segments, solution of the governing equation for each segment will calculated, and make a comparison of the total amount of mass generated with the mass discharge through the nozzle, if the check fails a new assumption is required to obtain a solution for one instant of time. The average burning rate is taken to be liner between the head and nozzle end.

Determination of head and nozzle end burning rate take into account erosive burning effect the burning surface at tail off is reduced in proportion to the length of the propellant that has experienced burn out according to the linear model of the regression surface. The computation are performed in two sections at the head and nozzle end of the grain, it is useful to for computing pressure, temperature, and velocity of gases of the nozzle end of the grain, the web thickness divided into equal segments to determine the output with minimum error.

We assume that the burning rate and pressure at head and nozzle end is linear along combustion

\section{Manufactures and Experiment of Modified SRM}

Modified two-inch motor consists of 3 segmented grains each one $108 \mathrm{~mm}$ by separating them by a small ring similar to the outer case each ring of width $10 \mathrm{~mm}$ and assembled together. The results will be shown later. The only modification of the modified 2'inch motor is to vary the length of the motor case; all the other dimensions are the same as the standard 2'inch motor. Also by changing the critical part of the nozzle we can get different pressure and different erosive ratio.

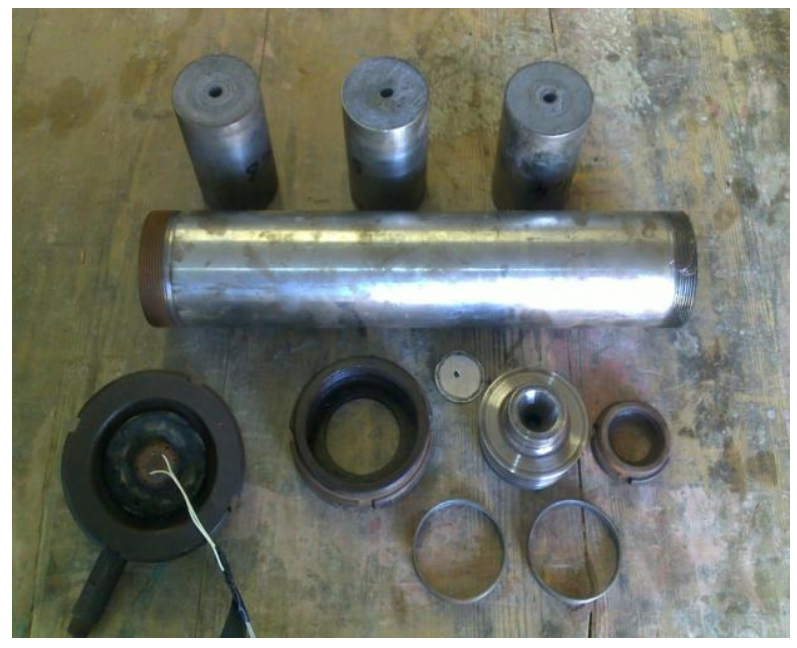

Figure 5. Parts of modified SRM.

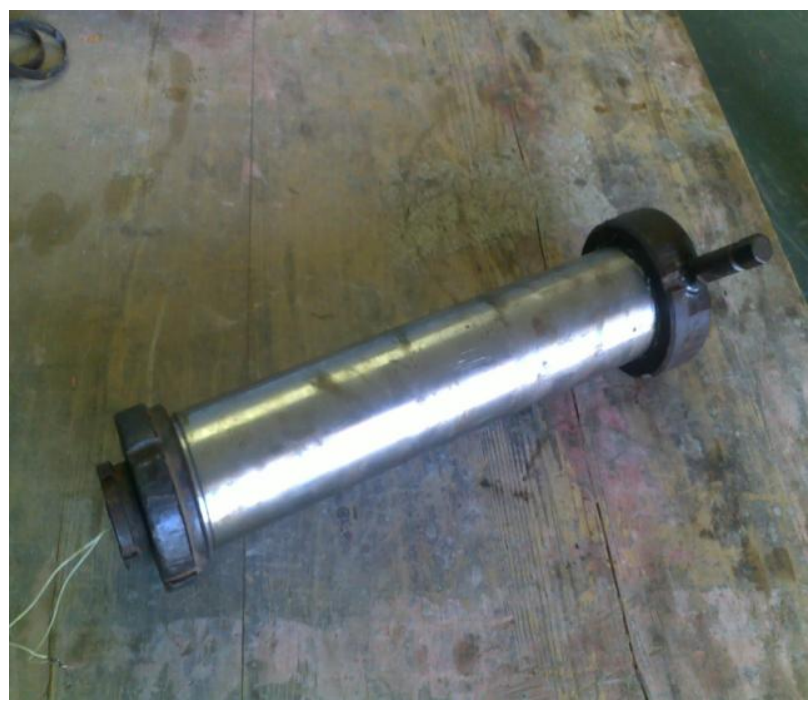

Figure 6. Assembled new motor.

\subsection{Experiment results}

The length of grain $=324 \mathrm{~mm}$ divided into 3 equal parts of solid propellant each one $108 \mathrm{~mm}$ separated by 2 steel rings each one of width $10 \mathrm{~mm}$, the outer diameter $=60$ $\mathrm{mm}$ and the inner diameter $=10 \mathrm{~mm}$, nozzle critical section $=9 \mathrm{~mm}$.

The pressure at the head end of the grain is measured experimentally while pressure at the nozzle end of the grain is predicted from the theoretical model.

Table 2. Results of modified two-inch motor

\begin{tabular}{|l|l|l|}
\hline D & $\mathrm{mm}$ & 10 \\
\hline Temp & $\mathrm{c}$ & 2 \\
\hline Throat & $\mathrm{mm}$ & 9 \\
\hline WT & $\mathrm{Kg}$ & 1.573 \\
\hline TD & Msec & 28 \\
\hline TB & Msec & 2.992 \\
\hline T50\% & Sec & 2.557 \\
\hline Pmax & Bar & 187 \\
\hline IPT & Bar.s & 334 \\
\hline Pav & Bar & 111 \\
\hline P50\% & Bar & 130 \\
\hline R50\% & $\mathrm{mm} / \mathrm{s}$ & 9.7 \\
\hline C star & $\mathrm{m} / \mathrm{sec}$ & 1352 \\
\hline
\end{tabular}




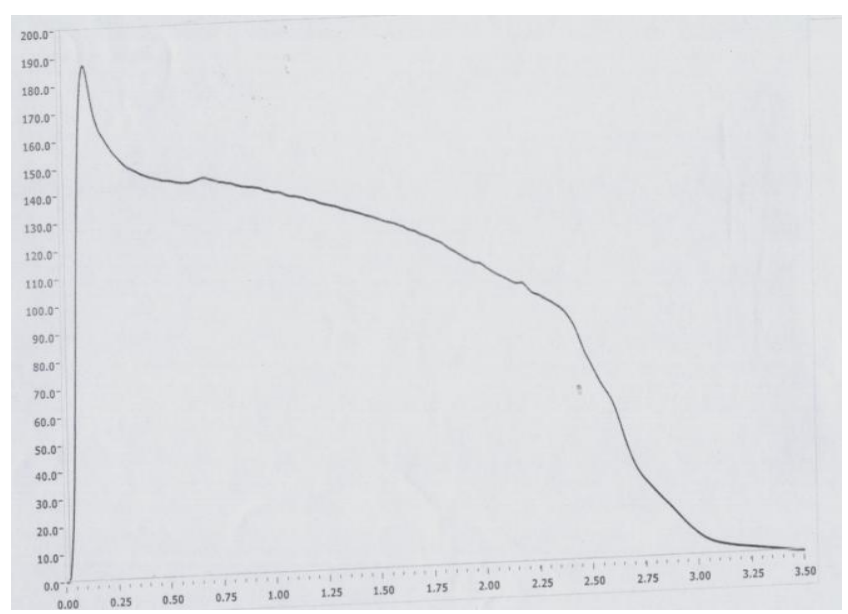

Figure 7. Pressure time curve of modified SRM.

All these curves are from the experiment sheet. In the beginning of the ignition the pressure increases in the small port area and the velocity of gases increased which causes erosive burning. This proves our Prediction and idea. The Very important conclusion could be drawn concerning the effect of very high pressure, very high velocity and the erosive burning. The critical section of the nozzle increases with respect to time, by measuring the critical section of the nozzle before and after burning, the critical section increase from 9 to $10.5 \mathrm{~mm}$. It is observed that increase of the critical area of about $25 \%$, which result in a drop in the pressure at the end of burning.

So there is a factor taken during calculation of the critical area which is the increase in the critical section with respect to time.

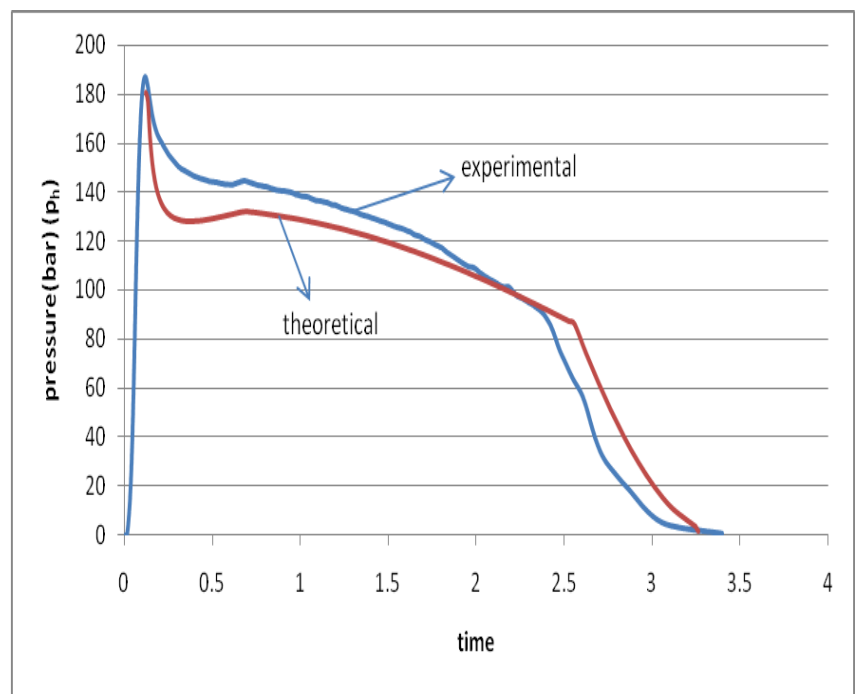

Figure 8. Experimental and theoretical pressure-time curves.

The assumption of the burning rate is taken to be linear along burning, so there is an error between the experimental and theoretical values.

The difference between the pressures at the head end and nozzle end of the grain is due to erosive burning, where at erosive the mass discharge is increased which causes this difference.

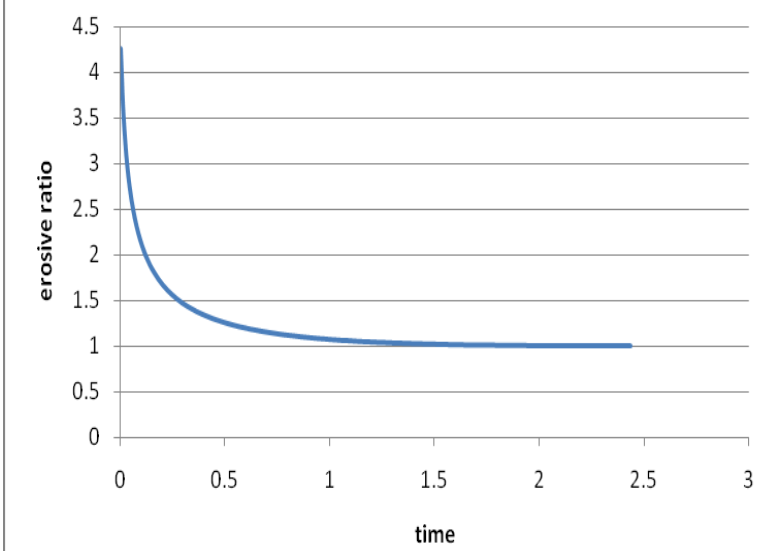

Figure 9. Erosive ratio versus time.

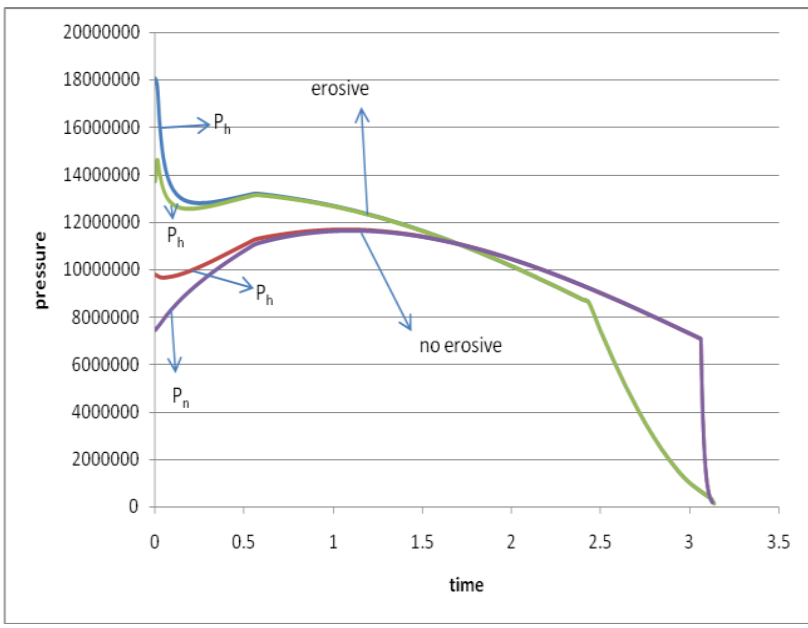

Figure 10. Pressure time cure with and without erosive burning.

\section{Conclusion}

The flow of combustion gaseous product at high velocity parallel to the surface of solid propellant is often found to lead to a significant increase in burning rate. This phenomenon is referred to as erosive burning and the increase in burning rate is known as the erosive burning rate. Studying the erosive burning is very important for better understanding of effects on the performance of the rocket motor. Good design of solid propellant rocket motor should account for possible erosive burning in order to thoroughly predict the pressure-time history and act accordingly for safe and best performance.

During the present study test rocket motors of standard and modified configurations were designed, manufactured and tested in different working conditions to investigate effects on the burning rate and their impact on the performance. In parallel, a theoretical model of the burning rate was developed and the proposed numerical solution was implemented as a computer code capable of predicting the behavior of the combustion pressure. In particular, the code treats the case of erosive burning.

The comparison between theoretical and experimental results showed fair agreements, which proves the validity 
of the code. The following results could be drawn from the present investigations:

By varying the grain length-to-diameter ratio or the critical diameter, in order to change the mass flux parallel to the surface, it was possible to generate experimentally and theoretically the condition of erosive burning. It was possible to predict the threshold velocity above which the erosive burning occurs. For a given type of propellant, the erosive ratio and threshold velocity are dependent on the combustion pressure. In the present study, it was found that with the higher pressure, lower erosive ratio, lower threshold velocity and higher threshold flux were encountered.

With smaller values of $(a, n)$ in the burning law, higher erosive ratios are encountered.

Some kind of oscillation occurs in the theoretical model. This may be attributed to the admitted values of the error considered in Mach number, mass balance and burning rate. More runs have been done for smaller values of permitted error between successive iterations and finally smooth curve could be reached. Clever choice of error values should be considered after several trials to get a smooth variation of producing curve.

The erosion at the nozzle critical section was measured and studied in the theoretical prediction. It has a significant effect on the combustion chamber pressure and consequently on the burning rate.

The code can be trusted for used in novel designs, and further investigations, saving numerous firing tests which are costly, time-consuming and risky

\section{References}

1. Barrere, "Rocket Propulsion," 1960.

2. O. B. Sutton P, "Rocket Propulsion Elements," 7 th edition, 2001.

3. J. W. KREIDLER, "Erosive Burning New Experimental Techniques and Methods of Analysis," solid propellant rocket conference, 1964.

4. M. K.King, "Erosive Burning of Composite Propellant," atlantic reserch coperation, 1976.

5. M. K. King, "Erosive Burning of Solid Propellants," JOURNAL OF PROPULSION AND POWER, vol. vol 9, 1993.

6. M. L. a. G.Robillard, "A Matimatical Method to Predict the Effects of Erosive Burning in Solid Rocket Propellant," 1957.

7. C. E. Rogers, "Erosive Burning Design Criteria For Solid Rocket Motor," NASSA article, 2002.

8. C. E. Rogers, "Solid Propellant Grain Design and Internal Ballistics,," NASSA report, vol. vol 33, 2004.

9. K. Srinivasan, S. Narayanan, and O. P. Sharma, "Numerical Studies on Erosive Burning in Cylindrical Solid Propellant Grain," Heat and Mass Transfer, vol. 44, pp. 579-585, 2007.

10. M. K. King, "Erosive Burning of Solid Propellants," journal of propullsion and power, vol. vol 9, 1993. 